\title{
MHD EQUILIBRIA OF HELICAL JETS
}

\author{
M. VILLATA AND A. FERRARI \\ Osservatorio Astronomico di Torino \\ Strada Osservatorio 20, I-10025 Pino Torinese (TO), Italy
}

\section{Introduction}

From recent high angular resolution $(\sim 0.1 \mathrm{arcsec})$ observations of some extragalactic jets (in particular we refer to the radio, optical, and ultraviolet images of the $\mathrm{M} 87$ jet, of $3 \mathrm{C} 66 \mathrm{~B}$, and $3 \mathrm{C} 264$ ) it can be inferred that the synchrotron emission from the jet comes mainly from an external, quasicylindrical surface on which bright helical filamentary structures can be clearly recognized.

A fundamental question that may arise in this connection is: how can the observed helical structures be in dynamical equilibrium? The starting point for such an investigation is a magnetohydrodynamic (MHD) description of the underlying physics. The observational evidence of helical morphologies suggesting that the outflow itself presents a helical symmetry urges towards the analytic study of helically symmetric MHD equilibria. The usefulness of this approach has been already stressed in Villata \& Tsinganos (1993) and Villata \& Ferrari (1994a,b, 1995). In particular, in the last two papers complete solutions of the whole set of time-independent MHD equations in helical symmetry were obtained and detailed models for the interpretation of helical jets (focusing the attention on the M 87 jet) constructed.

In this article we summarize some of the main final results.

\section{Main Results and Conclusions}

From the results obtained in this study we can conclude that the helical morphologies observed in some astrophysical jets can be interpreted starting from the MHD equilibrium of the underlying magnetized flow. In particular, by considering exact solutions of the time-independent MHD equations, one can construct models for the description of these peculiar structures. Under the assumption that the synchrotron emissivity is proportional to 


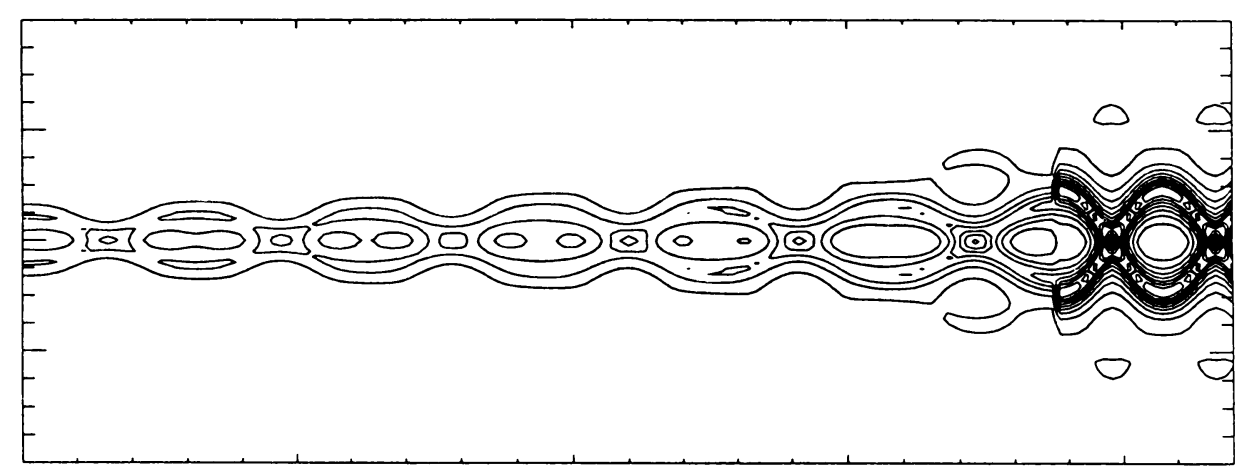

Figure 1. Subalfvénic model for the M 87 jet: equispaced contours of surface brightness

the plasma density $\rho$ and the magnetic field strength squared $B^{2}$, we can reproduce the observed high emission helical filaments by the combination of high $\rho$ and high $B^{2}$ filaments in conditions of MHD equilibrium.

Moreover, we have constructed specific models for the description of the main features of the M 87 jet: a double-stranded helical filamentary structure having an increasing pitch angle with increasing distance from the galaxy centre and an abrupt structural transition occurring in the outer part of the jet. A subalfvénic model (see Figure 1) seems to be more appropriate for the interpretation of the transition exhibited by the jet: it would be due to the interaction of the flow with the ambient medium at the epoch of the jet birth. The distribution of emission which comes out from this model is in good agreement with the observational one.

In conclusion, this study has provided (i) exact analytical solutions for helically symmetric (or in any other kind of symmetry with one ignorable coordinate) MHD equilibria and (ii) analytical models for the description of helical jets which for the first time allow to derive, by comparison with observations, (iii) the magnitudes of the relevant physical quantities (density, magnetic field, plasma velocity, etc.) by means of exact analytical formulae, thus providing (iv) a complete description of the system.

\section{References}

Villata, M. and Ferrari, A. (1994a) Exact Solutions for Helical MHD Equilibria of Astrophysical Jets, $A \mathscr{E} A$, Vol. no. 284, pp. 663-678

Villata, M. and Ferrari, A. (1994b) Exact Solutions for Helical Magnetohydrodynamic Equilibria. II. Nonstatic and Nonbarotropic Solutions, Phys. Plasmas, Vol. no. 1, pp. 2200-2206

Villata, M. and Ferrari, A. (1995) Exact Solutions for Helical MHD Equilibria of Astrophysical Jets. II. Non-barotropic Models, $A \& A$, Vol. no. 293, pp. 626-639

Villata, M. and Tsinganos, K. (1993) Exact Solutions for Helical Magnetohydrodynamic Equilibria, Phys. Fluids B, Vol. no. 5, pp. 2153-2164; Erratum (1994), Phys. Plasmas, Vol. no. 1, p. 216 